\title{
DA CONGRUÊNCIA DE VALOR ENTRE BOUNDARY SPANNERS À SATISFAÇÃO EM MERCADOS B2B: UMA PERSPECTIVA TEÓRICA
}

FROM VALUE CONGRUENCE BETWEEN BOUNDARY SPANNERS TO SATISFACTION IN B2B MARKETS: A THEORETICAL PERSPECTIVE

\section{DE LA CONGRUENCIA DE VALOR ENTRE BOUNDARY SPANNERS A LA SATISFACCIÓN EN MERCADOS B2B: UNA PERSPECTIVA TEÓRICA}

\author{
THAYANE WOELLNER SVIERCOSKI MANOSSO \\ Mestranda \\ Universidade de Passo Fundo - Brasil \\ thay thay3@hotmail.com \\ ORCID: https://orcid.org/0000-0002-9932-5520 \\ VERNER LUIS ANTONI \\ Doutor \\ Universidade de Passo Fundo - Brasil \\ antoni@upf.br \\ ORCID: https://orcid.org/0000-0002-7780-6470
}

Submetido em: 21/05/2018

Aprovado em: 28/09/2018

Doi: alcance.v25n2(Mai/Ago).p194-210

\section{RESUMO}

Os relacionamentos interorganizacionais dependem, entre outros fatores, das relações interpessoais desenvolvidas pelos agentes que atuam como representantes das empresas no mercado, os chamados boundary spanners. Como qualquer outro indivíduo, esses atores têm valores pessoais que influenciam seu comportamento e, por consequência, o desempenho da organização em que esse indivíduo atua. Por isso, selecionar parceiros comerciais que tenham valores semelhantes pode interferir no nível de satisfação em um relacionamento B2B. Com o propósito de assimilar essas relações, propõe-se, a partir de uma análise bibliométrica, um modelo de avaliação do impacto da congruência dos valores humanos dos boundary spanners na satisfação dos membros envolvidos em relações interorganizacionais. Como resultado e contribuição do estudo, são apontadas cinco hipóteses a serem testadas empiricamente em pesquisas futuras, apresentadas sinteticamente em um modelo teórico, com o intuito de corroborar os resultados.

Palavras- chave: Relacionamento B2B. Congruência de Valor. Satisfação.

\begin{abstract}
Interorganizational relationships depend, among other factors, on the interpersonal relations developed by the agents that act as representatives of companies in the market; the so-called boundary spanners. Like any other individual, these actors have personal values that influence their behavior and, consequently, the performance of the organization in which that individual works. Therefore, selecting business partners who have similar values may affect the level of satisfaction in a B2B relationship. In order to assimilate these relationships, a model is proposed, based on bibliometric analysis, that can be use to evaluate the impact of the congruence of human
\end{abstract}


values of boundary spanners in the satisfaction of members involved in interorganizational relationships. As a result and contribution of the study, five hypotheses are outlined, to be empirically tested in future research. These are presented synthetically, in a theoretical model, in order to corroborate the results.

Keywords: B2B relationship. Value Congruence. Satisfaction.

\section{RESUMEN}

Las relaciones interorganizacionales dependen de muchos factores, entre ellos, de las relaciones interpersonales desarrolladas por los agentes que actúan como representantes de las empresas en el mercado, los llamados boundary spanners. Como cualquier otro individuo, los boundary spanners tienen valores personales que influencian su comportamiento y, por consiguiente, el desempeño de la organización en que ese individuo actúa. Por eso, seleccionar socios comerciales que tengan valores similares puede interferir en el nivel de satisfacción en una relación B2B. Con el propósito de asimilar esas relaciones, se propone, a partir de un análisis bibliométrico, un modelo de evaluación del impacto de la congruencia de los valores humanos de los boundary spanners en la satisfacción de los miembros involucrados en relaciones interorganizacionales. Como resultado y contribución del estudio, se indican cinco hipótesis a ser probadas empíricamente en investigaciones futuras, presentadas sintéticamente en un modelo teórico, con el fin de corroborar los resultados.

Palabras clave: Relaciones B2B. Congruencia de valor. Satisfacción.

\section{INTRODUÇÃO}

No marketing tradicional, a solução para o cliente é um produto na forma de um bem físico ou de um serviço; enquanto no marketing de relacionamento, a solução é o próprio relacionamento (Grönroos, 2004). Diante disso, as expectativas de uma recompensa não econômica, psicológica e social pode tornar-se mais importante do que os retornos econômicos em relações interorganizacionais (Paswan, Blankson \& Guzman, 2011).

Apesar do número de pesquisas voltadas ao mercado Business-to-Business (B2B) ainda ser muito baixo quando comparado ao mercado Business-to-Consumer (B2C) (Lilien, 2016), a atenção que os relacionamentos B2B passaram a receber nos últimos anos fez com que os agentes responsáveis pelo marketing expandissem suas ações na empresa (Valério, 2015), incluindo qualquer funcionário que interagisse direta ou indiretamente com os clientes ou que participasse da tomada de decisão de compra (Grewal et al., 2015).

Nesse sentido, uma das dimensões relevantes no estudo de relacionamentos B2B é o contexto micro, em que pesquisadores investigam como características de membros de organizações impactam no funcionamento e nos resultados do relacionamento entre empresas (Cropper, Ebers, Huxham \& Ring, 2014). Um dos atributos contextuais de micronivel inclui a ligação entre os boundary spanners, atores que fazem a ponte entre duas organizações (Seabright, Levinthal \& Fichman, 1992; Beechler, Sondergaard, Miller \& Bird, 2004; Chakkol, Karatzas, Johnson \& Godsell, 2018).

Para que se possa entender melhor o relacionamento B2B, é fundamental examinar as relações interpessoais dos boundary spanners em termos de suas conexões sociais (Leung, Lai, Chan \& Wong, 2005; Wang, Kang, Childerhouse \& Huo, 2018), já que esses atores representam o meio mais importante na construção e na manutenção de relacionamentos sólidos entre empresas (Larentis, Antonello \& Slongo, 2018). Apesar da importância desses atores, poucos estudos avaliaram o seu impacto no relacionamento em mercados B2B (Dong, Ma \& Zhou, 2017).

Nessa perspectiva, esses atores, como quaisquer outros, podem ter seus próprios objetivos e experiências, afetando seu comportamento e desempenho dentro da empresa, o que acaba por interferir nos relacionamentos interorganizacionais (Håkansson, 1982). É importante, portanto, entender as características psicológicas envolvidas nas relações B2B, em especial as predisposições, estrutura de preferências e modelo de decisão dos atores, que servem como base para as decisões estratégicas da empresa (Webster \& Wind, 1996).

Entre os aspectos pessoais que podem influenciar o comportamento humano, estão os valores humanos (Tamayo, 2005), que, ao se articularem entre si, caracterizam um sistema de valores estável e sólido, o que pode ser um desafio em relacionamentos B2B (Rokeach, 1973; Schwartz, 1992). Pesquisas permitem afirmar que a 
escolha por um parceiro com valores semelhantes é o que pode ditar a cooperação a longo prazo em relacionamentos entre empresas (Wang \& Zhang, 2017; Larentis et al., 2018).

Nessa mesma linha de raciocínio, Wang e Zhang (2017) propuseram que os valores possam ser um outro método de governança relacional (Heide \& John, 1992). No entanto, é necessário o avanço nessa via de pesquisa, já que o conhecimento sobre a congruência de valores e seus efeitos em relacionamentos B2B ainda são muito limitados na disciplina de marketing (Zhang \& Bloemer, 2008; Kashyap \& Sivadas, 2012; Zhu \& Chang, 2013; González-Rodríguez, Díaz-Fernández \& Simonetti, 2015; Wang \& Zhang, 2017).

A teoria da semelhança-atração, da psicologia social, indica que as pessoas têm maiores intenções de criar e manter suas relações com aqueles que são semelhantes a si próprios (Gaunt, 2006). Para Schein (2010), os valores constituem um elemento fundamental da cultura organizacional, impactando tanto o desempenho individual quanto o organizacional. Da mesma forma, em abordagens sociológicas, os valores já são tratados como um aspecto da cultura e estudados pelas suas influências nos indivíduos (Cieciuch, Schwartz \& Davidov, 2015).

A teoria da troca social já pregava que valores congruentes entre parceiros fornecem uma base para a cooperação e um relacionamento sustentável (Blau, 1964). Ainda, segundo Larentis e Slongo (2008), relacionamentos entre empresas desenvolvem-se mais rapidamente quando as partes possuem, entre outras características, valores e crenças semelhantes. No entanto, "os pesquisadores de marketing de relacionamento devem prestar mais atenção à congruência de valor" (Zhang \& Bloemer, 2008, p. 12), visto que o potencial de governança dos valores nas trocas B2B é ainda pouco explorado (Wang \& Zhang, 2017).

Muitos pesquisadores avaliaram a congruência de valores entre funcionários e organizações (Byza, Dörr, Schuh \& Maier, 2017; Lee et al., 2017), entre funcionários e seus líderes (Qu, Dasborough, Zhou \& Todorova, 2017; Lajoie, Boudrias, Rousseau \& Brunelle, 2017; Jensen, 2018) e da perspectiva de uma das empresas envolvidas no relacionamento (Kashyap \& Sivadas, 2012; Sabbir \& Nazrul, 2014; Wang \& Zhang, 2017; He, Huang \& Wu, 2018), mas não foram encontrados, a partir da pesquisa bibliométrica realizada, estudos que identifiquem os valores humanos pessoais dos boundary spanners em ambos os lados da díade em canais de marketing.

Ainda assim, sabe-se que o objetivo comum dos relacionamentos B2B é estabelecer relações duradouras com seus clientes (Dwyer, 1980). Segundo Lussier e Hartmann (2017), a satisfação dos membros envolvidos, a qual depende de aspectos psicológicos, é a chave para a viabilidade do relacionamento a longo prazo (Geyskens, Steenkamp \& Kumar, 1999; Mpinganjira, Roberts-Lombard \& Svensson, 2017). A conquista da satisfação nas relações comprador-vendedor depende da presença de algum nível de congruência entre os parceiros (Mehta, Larsen, Rosenbloom \& Ganitsky, 2006), o que os torna menos propensos a sair de um relacionamento (Voldnes, Grønhaug \& Nilssen, 2012).

Essa discussão teórica aponta o mérito de se entender e gerenciar os relacionamentos interpessoais, intrínsecos aos relacionamentos interorganizacionais, quando se busca a fidelização de clientes por meio da satisfação. O crescente interesse do mercado pelos relacionamentos B2B (Valério, 2015; Watson, Worm, Palmatier \& Ganesan, 2015) ainda não se reflete no número de pesquisas publicadas sobre o tema (Lilien, 2016), o que remete à importância de construir-se uma ponte entre os problemas dos profissionais e os esforços da academia, buscando o aumento de equilibrio no dilema de rigor versus relevância no marketing (Cortez \& Johnston, 2017).

Diante disso, o presente artigo, de cunho teórico, tem como objetivo propor um modelo para a avaliação do impacto da congruência dos valores humanos dos boundary spanners na satisfação dos membros envolvidos em relacionamentos $\mathrm{B} 2 \mathrm{~B}$, contribuindo com a temática ao apresentar as hipóteses desenvolvidas a partir de lacunas teóricas propostas por autores de alta relevância, tanto da disciplina de marketing relacional, quanto de relacionamento $\mathrm{B} 2 \mathrm{~B}$.

Para tanto, o artigo expõe, em sua apresentação, a importância e os gaps relacionados aos temas. Por se tratar de um estudo teórico, a sessão seguinte esclarece o método utilizado para a construção do referencial teórico, o qual é apresentado na sequência, e dá base às hipóteses desenvolvidas. Em seguida são apresentados os resultados, tendo como foco o detalhamento do modelo desenvolvido e, por fim, as considerações finais. 


\section{MÉTODO}

Para o desenvolvimento do presente estudo, definiu-se como técnicas de pesquisa teórica a bibliometria e o levantamento bibliográfico, o que torna possível mensurar e compreender o conhecimento de determinada área científica (Teixeira, Iwamoto \& Medeiros, 2013). A mensuração, aliada à compreensão contextualizada da produção científica e de seus autores (Splitter, Rosa \& Borba, 2012), possibilita estabelecer uma parceria entre técnicas bibliométricas e abordagens teóricas (Araújo, 2002).

A pesquisa teve seu início na busca pelo estado da arte do conhecimento em relacionamentos B2B. Nesta etapa, utilizaram-se como critério de busca apenas artigos publicados em Journals, durante o período de 20142018, na área de Business, Management and accounting. Foram avaliados 91 artigos, nacionais e internacionais, recuperados a partir das bases Scopus, Web of Science, Science Direct, Google Acadêmico, Spell e Scielo.

Os artigos foram lidos por completo e classificados em diferentes grupos, dependendo do objetivo proposto por cada um. 0 grupo tomado como base para a evolução da pesquisa foi o de artigos teóricos, que possuíam em sua essência a finalidade de propor estudos futuros em relacionamentos interorganizacionais, como a relação entre indivíduos e organizações (Watson et al., 2015), a venda pessoal como vantagem competitiva (Lilien, 2016), o vínculo entre comprador e vendedor e como ele pode ser medido (Cortez \& Johnston, 2017), entre outros.

O estudo de Wang e Zhang (2017), em um dos 91 artigos analisados, deu início ao desenvolvimento do modelo, por apontar diversas lacunas teóricas relacionadas às apontadas pelos autores de artigos teóricos, como a identificação de valores específicos compartilhados entre empresas, a coleta de dados diádicos e o papel dos valores pessoais no relacionamento entre empresas (Wang \& Zhang, 2017). A partir das sugestões dos autores, foram realizados consecutivos levantamentos bibliográficos sobre os temas propostos.

Além de Wang e Zhang (2017), outros autores abordaram a congruência de valores, como Zhang e Bloemer (2008), que exploraram a influência da congruência de valores pessoais sobre aspectos do relacionamento em contextos B2C e, para tanto, utilizaram a escala de valores de Schwartz (1992). Por meio de novo levantamento teórico, identificou-se que Schwartz se destaca na pesquisa de valores pessoais (Eva, Prajogo \& Cooper, 2017; Ye, Soutar, Sneddon \& Lee, 2017), e que a escala mais atual proposta pelo autor foi validada no Brasil por Torres et al. (2016).

Para tornar viável a pesquisa de valores pessoais no contexto B2B, é necessário compreender os atores envolvidos nos relacionamentos interpessoais, intrínsecos aos relacionamentos interorganizacionais, já que estes são os detentores dos valores congruentes em uma troca comercial (Dong et al., 2017; Lussier \& Hall, 2018). 0 estudo de Larentis et al. (2018) destaca a importância dos boundary spanners nos relacionamentos interorganizacionais e sua influência no desenvolvimento de uma cultura de fronteiras.

Por fim, como indicador de sucesso de relacionamentos entre empresas, a satisfação se mostrou uma variável importante, segundo os estudos analisados, por ser considerada uma filosofia orientadora das relações comerciais (Yeung, Ramasamy, Chen \& Paliwoda, 2013). Nesse sentido, os estudos de Geyskens e Steenkamp (2000), Del Bosque Rodríguez et al. (2006), Ha et al. (2016) e Mpinganjira et al. (2017) mostram importantes evoluções e adaptações nas escalas de satisfação, tanto econômica quanto não econômica. A Figura 1 ilustra 0 design teórico da construção do modelo de pesquisa. 


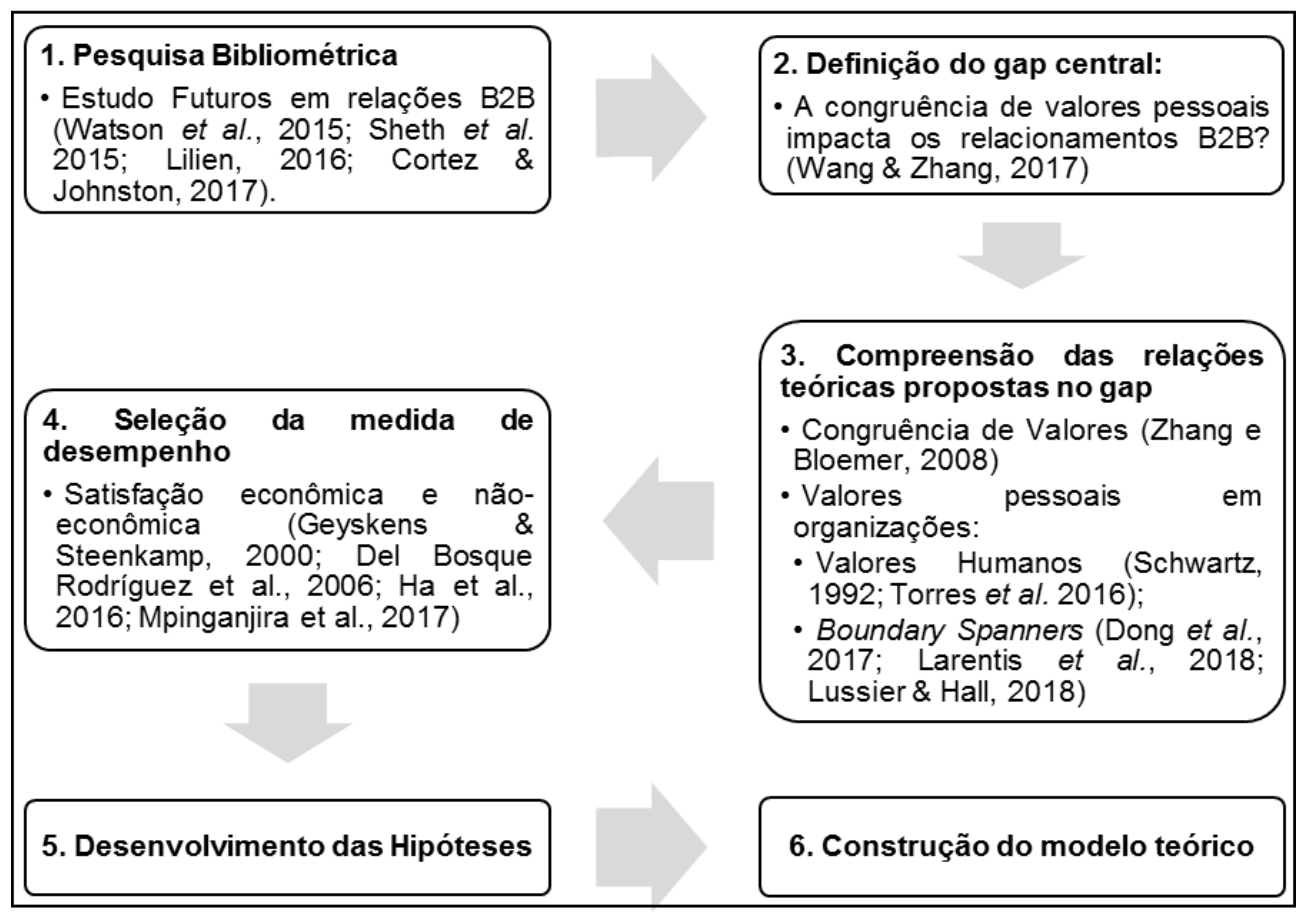

Figura 1: Design teórico da pesquisa Fonte: Elaborada pelos autores.

Com a definição dos temas a serem incluídos no modelo e a compreensão da relação estabelecida entre estes, entendeu-se também como relevante a permanência de alguns autores seminais, seja pelo pioneirismo e/ou destaque de seus modelos na literatura de B2B (Schwartz, 1992; Shani \& Chalasani, 1992; Morgan \& Hunt, 1994; Geyskens et al., 1999; Farrelly \& Quester, 2005; Ramaseshan, Yip \& Pae, 2006; Lai, 2007; Schwartz et al., 2012; Sivadas, Holmes \& Dwyer, 2012), seja pelo prévio estabelecimento de relações entre os temas em questão (Geyskens \& Steenkamp, 2000; Abdul-Muhmin, 2005; Ferguson, Paulin \& Bergeron, 2005; Del Bosque Rodríguez, Agudo \& San Martín Gutiérrez, 2006; Lian \& Laing, 2007; Zhang \& Bloemer, 2008; Ellegaard, 2012; Kashyap \& Sivadas, 2012; Voldnes et al., 2012).

\section{REFERENCIAL TEÓRICO E APRESENTAÇÃO DAS HIPÓTESES}

Os primeiros modelos sobre como as empresas realizam negócios entre si e a teoria sobre 0 comportamento de compra organizacional (Webster \& Wind, 1996) tiveram suas raízes, principalmente, no marketing de consumo (Johnsen, Lamming \& Harland, 2014). A partir disso, a compra repetida e a fidelidade à marca começaram a ganhar destaque na literatura de marketing (Sheth, Parvatiyar \& Sinha, 2015). Até hoje, na atual era de hipercompetição, os profissionais de marketing acreditam que a retenção e a fidelidade de clientes são menos dispendiosas e talvez uma vantagem competitiva mais sustentável do que a aquisição de novos clientes, tanto em mercados B2C como B2B (Sheth et al., 2015).

Embora os relacionamentos sejam essenciais em ambos os mercados para a retenção e a fidelização de clientes, nas configurações B2B, especialmente, o relacionamento com clientes demora mais para se estabelecer, é mais longo, exibe custos de troca mais altos e tem impactos maiores nos resultados do que nos mercados B2C (Zhang, Netzer \& Ansari, 2014; Zhang et al., 2016), o que torna o gerenciamento dos relacionamentos interorganizacionais especialmente críticos (Harmeling \& Palmatier, 2015; Cortez \& Johnston, 2017).

Segundo Lilien (2016), essas relações comerciais podem ser estudadas por meio de gerentes, de um centro de compras inteiro, de toda uma unidade de negócios estratégica, de uma relação entre empresas ou mesmo de uma rede. Assim, as estruturas e os métodos teóricos em uma pesquisa B2B particular podem variar desde teorias como a RBV até as teorias psicológicas (Lilien, 2016).

Webster e Wind (1996) já consideravam que a tomada de decisão em compras organizacionais é influenciada por diversos fatores, que podem ser agrupados em ambientais, organizacionais, sociais e individuais. 
Especificamente no que se refere aos fatores individuais, destaca-se que são pessoas, e não organizações, que tomam as decisões de compra (Webster \& Keller, 2004). Ou seja, a autoridade decisória final ainda reside tipicamente em um único indivíduo ou pequeno grupo executivo (Grewal et al., 2015) e, por isso, as normas ou as expectativas de comportamento são geradas, frequentemente, por meio das interações entre os atores que constituem um canal (Coughlan, Anderson, Stern \& El-Ansary, 2012), o que normalmente é tarefa dos boundary spanners.

O boundary spanner, que pode ser um vendedor, um comprador ou um gerente que atua em relacionamentos interorganizacionais, é também um representante da organização ou intérprete do ambiente externo em fronteiras organizacionais (Araujo, Dubois \& Gadde, 2003). Estudiosos afirmam que o relacionamento interpessoal entre os boundary spanners é um fator essencial na gestão de canais de marketing orientados para o relacionamento e deve ser um laço incorporado nas relações B2B (Zhuang \& Zhang, 2011; Dong et al., 2017). Além disso, o estabelecimento e a manutenção de relações organizacionais dependem dos boundary spanners, já que esses indivíduos interagem uns com os outros por meio dos limites organizacionais (Ellegaard, 2012).

Segundo Andrade e Leite (2017), o comportamento e as atitudes dos indivíduos têm sido estudados por filósofos (Pavlov \& Gantt, 1941; Fula, 2004; Rein \& Eysenck, 2018), cientistas sociais (Ajzen, 1971; Lyons, Wodarski \& Feit, 1998; Lebbon \& Sigurdsson, 2017) e psicólogos (Woodruff, 1949; Eagly \& Wood, 1999; Torres et al., 2016), na busca pela compreensão dos elementos fundamentais que os determinam. Nesse sentido, dentre as correntes teóricas seguidas por estudiosos do marketing, a Teoria dos Valores Humanos Pessoais se mostra uma importante vertente no entendimento de comportamentos (Thøgersen \& Olander, 2002; Tamayo, 2005; Torres et al., 2016). Atualmente, existem mais de uma dezena de instrumentos de mensuração de valores pessoais, e a escolha de uma delas se justifica pelo contexto da pesquisa em questão (Andrade \& Leite, 2017).

Segundo Sambiase, Teixeira, Bilsky, Araujo \& Domenico (2014), um dos primeiros e mais importantes estudiosos da Teoria dos Valores Pessoais foi o psicólogo Milton Rokeach. Influenciado por Allport e amparado pela psicologia social, Rokeach (1973) desenvolveu o primeiro instrumento a usar uma escala ordinal, o Rokeach Values Survey (RVS), dividindo os valores em Terminais e Instrumentais, hierarquizados como princípios-guia na vida dos respondentes (Madi, 2016). À luz da teoria de Rokeach (1973), Schwartz e Bilsky (1987) iniciaram seus estudos, supondo que não houvesse uma separação dos valores em terminais e instrumentais, conforme propunha Rokeach (Sambiase et al., 2014). A dúvida se confirmou, e com isso os autores apresentaram, em 1992, a Schwartz Value Survey (SVS) (Schwartz, 1992). Na busca de um instrumento mais parcimonioso que continuasse a representar a teoria de valores básicos desenvolvida, Schwartz et al. (2001) criaram o Portrait Values Questionnaire (PVQ), constituído por 40 itens e validado em diversos países, inclusive no Brasil (Schwartz, 2005; Porto, 2005; Tamayo, 2007).

Visando ampliar a capacidade heurística e o poder explanatório da teoria (Sambiase et al., 2014), Schwartz et al. (2012), apresentaram a versão refinada e um novo instrumento de mensuração similar às proposições anteriores, o PVQ-Refined (PVQ-RR), que se baseia na ideia de um continuum motivacional dividido em um conjunto mais estreito de valores. O estudo de Torres et al. (2016) traduziu e validou o PVQ-RR no Brasil, além de testar a utilidade e a validade do poder preditivo dos 19 valores para comportamentos - uma vez que, em estudos anteriores, Schwartz et al. (2012) fizeram esta associação com atitudes e crenças, mas não com comportamentos (Vilela, 2017).

Em meio às razões que justificam o interesse do marketing pelos valores humanos pessoais, destacamse suas capacidades de explicar e predizer comportamentos em relação aos modos de conduta dos indivíduos perante a sociedade (Schwartz et al., 2012; Andrade \& Leite, 2017). Os valores são considerados pelas abordagens sociológicas como um aspecto da cultura que, por sua vez, é capaz de influenciar valores, crenças, normas e comportamentos das pessoas (Leung et al., 2005; Cieciuch et al., 2015). Como as diferenças culturais entre parceiros comerciais podem resultar em mal-entendidos, devido à falta de um quadro de referência compartilhado, a compreensão do grau em que os valores são congruentes entre os membros de uma relação B2B torna-se relevante (Voldnes et al., 2012).

A congruência de valores em relacionamentos interorganizacionais refere-se à "medida em que os parceiros têm crenças em comum sobre quais comportamentos, metas e políticas são importantes ou não importantes, apropriados ou inadequados, certos ou errados" (Morgan \& Hunt, 1994, p. 25) e está enraizada no sistema de crenças fundamentais de uma empresa, em que os valores são prescrições de como os participantes devem se comportar (Wang \& Zhang, 2017). 
Assim, estudiosos de marketing sugerem que a congruência de valor entre os parceiros de troca controla e racionaliza as tomadas de decisões (Enz, 1988), gera entendimentos mútuos e expectativas comuns (Moorman, Zaltman, \& Deshpandé, 1992), promove confiança (Sitkin \& Roth, 1993), influencia no desejo de seguirem os acordos iniciais (Murry \& Heide, 1998), sustenta a harmonia de interesses, o que reduz a probabilidade de comportamento oportunista (Ren, 2010), ajuda a desenvolver normas e diretrizes de ambos os parceiros (Schein, 2010), direciona os objetivos e os comportamentos para atingi-los (Malbašić, Rey, \& Potočan, 2015), reforça a coordenação e o desempenho por meio de metas comuns internalizadas (Kashyap \& Sivadas, 2012) e evita futuras violações de expectativas (Wang \& Zhang, 2017).

A congruência entre valores também é pré-requisito na seleção de parceiros, mesmo que intrinsicamente, e antecedente indispensável da coordenação, da cooperação, do compromisso, da confiança, do desempenho e da própria cultura organizacional (Blau, 1964; Dwyer, Schurr \& Oh, 1987; Enz, 1988; Heide \& John, 1992; Moorman et al., 1992; Sitkin \& Roth, 1993; Morgan \& Hunt, 1994; Murry \& Heide, 1998; Zhang \& Bloemer, 2008; Kashyap \& Sivadas, 2012; Sabbir \& Nazrul, 2014; Zhang et al., 2016; Cazier, Shao \& Louis, 2017; Wang \& Zhang, 2017; Larentis et al., 2018). Diante do exposto, pode-se supor que:

\section{H1. Existe congruência positiva e significativa entre os valores humanos pessoais dos boundary spanners das empresas envolvidas no relacionamento B2B.}

Segundo Zhang e Bloemer (2008), os compradores com baixa congruência de valor podem se sentir satisfeitos com a compra uma vez, mas é menos provável que mantenham e aprimorem seu relacionamento com o fornecedor apenas para atingir seus valores. Além disso, a maioria das pesquisas B2B concentra-se na presença ou na ausência de satisfação no relacionamento, ignorando como, por que e para que os parceiros desenvolvem esse julgamento de satisfação (Kashyap \& Sivadas, 2012). Estudos sugerem que existe uma relação entre valores congruentes de parceiros B2B e a satisfação no relacionamento (Geyskens et al., 1999; Abdul-Muhmin, 2005; Zhang \& Bloemer, 2008; Kashyap \& Sivadas, 2012; Voldnes et al., 2012; Mpinganjira et al., 2017), o que permite afirmar que:

\section{H2. Existe uma relação significativa entre a congruência de valores dos boundary spanners e a satisfação geral das empresas envolvidas no relacionamento B2B.}

A satisfação pode ser definida como um estado afetivo positivo resultante de uma avaliação de todos os aspectos da relação entre empresas (Dwyer et al., 1987; Abdul-Muhmin, 2005; Voldnes et al., 2012). Segundo Ring e Van de Ven (1994), o desejo de negociar novamente com a contraparte é decorrente majoritariamente da satisfação com negociações anteriores.

Empiricamente, a importância da satisfação é evidenciada pelos muitos estudos que encontraram satisfação como um fator importante para o sucesso das relações em mercados B2B (Del Bosque Rodríguez et al., 2006; Human \& Naudé, 2014; Hashim \& Tan, 2015) e pelos autores que provaram a existência de relações diretas e indiretas entre a satisfação, a confiança e o comprometimento em relações interorganizacionais (Svensson, Mysen \& Payan, 2010; Voldnes et al., 2012; Mpinganjira, Bogaards, Svensson \& Mysen, 2014; Sarmento, Simões \& Farhangmehr, 2014; Hashim \& Tan, 2015; Mpinganjira et al., 2017).

A maioria dos pesquisadores trata a satisfação como uma variável multifacetada. Muitos concordam que uma das dimensões é econômica, porém existe uma corrente que define a segunda dimensão como não econômica (Farrelly \& Quester, 2005; Ferro et al., 2016; Mpinganjira et al., 2017) e outra como social (Ramaseshan et al., 2006; Lai, 2007; Geyskens \& Steenkamp, 2000; Ha et al., 2016). Assim, nas hipóteses que seguem, propõese que:

H2a. Existe uma relação significativa entre a congruência de valores dos boundary spanners e a satisfação econômica das empresas envolvidas no relacionamento B2B.

H2b. Existe uma relação significativa entre a congruência de valores dos boundary spanners e a satisfação não econômica ou social das empresas envolvidas no relacionamento B2B.

Além de testar a natureza plural da satisfação, muitos pesquisadores do tema recomendam estudos que identifiquem a presença ou não de uma relação direta entre a satisfação econômica e não econômica ou social, já que alguns autores encontraram resultados em que a primeira antecede a segunda (Ferro et al., 2016; Sivadas et al., 2012; Mpinganjira et al., 2017), e outros em que a satisfação não econômica ou social é que prediz a satisfação econômica (Farrelly \& Quester, 2005; Lai, 2007). Como não se sabe ao certo se há uma relação entre elas e, em caso positivo, qual a precursora e qual a resultante, supõe-se apenas que: 
H3. Existe uma relação significativa entre a satisfação econômica e a satisfação não econômica ou social das empresas envolvidas no relacionamento B2B.

Diante do exposto, percebem-se a importância e a carência de estudos que unam temas como os valores pessoais, bem como a congruência destes entre indivíduos e o contexto interorganizacional, com o objetivo de entender se a satisfação com o relacionamento B2B possui relação com a congruência de valores entre os boundary spanners envolvidos em relações interpessoais.

\section{ANÁLISE E DISCUSSÃO DOS RESULTADOS}

As hipóteses apresentadas anteriormente têm como objetivo 0 avanço na teoria sobre as influências pessoais em relacionamentos B2B e, por isso, devem ser validadas empiricamente. Como forma de elucidação e sintetização do que foi apontado, a Figura 2 expõe as hipóteses formuladas, os temas potencialmente importantes na construção de cada uma delas e suas respectivas fundamentações teóricas.

\begin{tabular}{|c|c|c|c|}
\hline $\mathrm{H}$. & Descrição & Temas & Fundamentação teórica \\
\hline \multirow{5}{*}{$\mathrm{H} 1$} & \multirow{5}{*}{$\begin{array}{l}\text { Existe congruência positiva e } \\
\text { significativa entre os valores } \\
\text { humanos pessoais dos boundary } \\
\text { spanners das empresas envolvidas } \\
\text { no relacionamento B2B. }\end{array}$} & $\begin{array}{c}\text { Marketing de } \\
\text { Relacionamento }\end{array}$ & $\begin{array}{c}\text { Shani e Chalasani (1992); Morgan e Hunt } \\
\text { (1994); Sheth et al. (2015) }\end{array}$ \\
\hline & & $\begin{array}{l}\text { Relacionamento } \\
\text { B2B }\end{array}$ & $\begin{array}{l}\text { Watson et al. (2015); Lilien (2016); Zhang } \\
\text { et al. (2016); Cortez e Johnston (2017); }\end{array}$ \\
\hline & & $\begin{array}{l}\text { Boundary } \\
\text { Spanners }\end{array}$ & $\begin{array}{c}\text { Ferguson et al. (2005); Lian e Laing } \\
\text { (2007); Ellegaard (2012); Dong et al. } \\
\text { (2017); Larentis et al. (2018); Lussier e } \\
\text { Hall (2018) } \\
\end{array}$ \\
\hline & & $\begin{array}{l}\text { Valores humanos } \\
\text { pessoais }\end{array}$ & $\begin{array}{c}\text { Schwartz (1992); Schwartz et al. (2012); } \\
\text { Grueso-Hinestroza e Antón- Rubio } \\
\text { (2015); Torres et al. (2016) }\end{array}$ \\
\hline & & $\begin{array}{l}\text { Congruência de } \\
\text { Valores }\end{array}$ & $\begin{array}{l}\text { Zhang e Bloemer (2008); Kashyap e } \\
\text { Sivadas (2012); Wang e Zhang (2017) }\end{array}$ \\
\hline $\mathrm{H} 2$ & $\begin{array}{l}\text { Existe uma relação significativa } \\
\text { entre a congruência de valores dos } \\
\text { boundary spanners e a satisfação } \\
\text { geral das empresas envolvidas no } \\
\text { relacionamento B2B. }\end{array}$ & \multirow{3}{*}{$\begin{array}{l}\text { Satisfação em } \\
\text { contextos B2B }\end{array}$} & \multirow{3}{*}{$\begin{array}{l}\text { Geyskens et al. (1999); Abdul-Muhmin } \\
\text { (2005); Farrelly e Quester (2005); } \\
\text { Ramaseshan et al. (2006); Lai (2007); } \\
\text { Sivadas et al. (2012); Voldnes et al. } \\
\text { (2012); Dadzie (2015) }\end{array}$} \\
\hline $\mathrm{H} 2 \mathrm{a}$ & $\begin{array}{l}\text { Existe uma relação significativa } \\
\text { entre a congruência de valores dos } \\
\text { boundary spanners e a satisfação } \\
\text { econômica das empresas } \\
\text { envolvidas no relacionamento B2B. }\end{array}$ & & \\
\hline $\mathrm{H} 2 \mathrm{~b}$ & $\begin{array}{l}\text { Existe uma relação significativa } \\
\text { entre a congruência de valores dos } \\
\text { boundary spanners e a satisfação } \\
\text { não econômica ou social das } \\
\text { empresas envolvidas no } \\
\text { relacionamento B2B. }\end{array}$ & & \\
\hline $\mathrm{H} 3$ & $\begin{array}{l}\text { Existe uma relação significativa } \\
\text { entre a satisfação econômica e a } \\
\text { satisfação não econômica ou social } \\
\text { das empresas envolvidas no } \\
\text { relacionamento B2B. }\end{array}$ & $\begin{array}{l}\text { Satisfação } \\
\text { econômica vs. } \\
\text { Não econômica } \\
\text { ou social em } \\
\text { contextos B2B } \\
\end{array}$ & $\begin{array}{c}\text { Geyskens e Steenkamp (2000); Del } \\
\text { Bosque Rodríguez et al. (2006); Ferro et } \\
\text { al. (2016); Ha et al. (2016); Mpinganjira et } \\
\text { al. (2017) }\end{array}$ \\
\hline
\end{tabular}

Figura 2: Hipóteses propostas, descrições, temas relevantes e respectivas fundamentações teóricas Fonte: Elaborado pelos autores. 
A formulação da primeira hipótese, que por meio da teoria supõe que existe congruência entre os valores humanos pessoais dos boundary spanners envolvidos nos relacionamentos interorganizacionais, teve seu início nas pesquisas em marketing relacional. Os conceitos de marketing de relacionamento envolvem o esforço integrado nas atividades de estabelecer, manter e melhorar relações com clientes e outros parceiros, com 0 objetivo de gerar benefícios de maior valor econômico, social e psicológico (Shani \& Chalasani, 1992; Morgan \& Hunt, 1994; Sheth et al., 2015). Ressaltam-se a importância e a relevância do estudo de Sheth et al. (2015), que destaca a necessidade de melhorar a identificação de construtos preditores de sucesso em relacionamentos B2B.

A partir do conceito de marketing de relacionamento, destaca-se que desenvolver relações mais próximas com os clientes e transformá-los em leais são aspectos tão importantes quanto atrair novos clientes (Berry, 1983). Essa característica se torna muito mais relevante em mercados B2B do que em B2C, já que, na primeira, as relações com compradores empresariais são mais difíceis de se estabelecer e impactam os resultados de forma mais intensa (Zhang et al., 2014; Zhang et al., 2016; Cortez \& Johnston, 2017).

Para Lilien (2016), podem ser consideradas relações B2B aquelas entre (i) fabricantes, atacadistas e varejistas; (ii) empresas farmacêuticas, hospitais e médicos; e (iii) empresas do agronegócio e agricultores e, para Watson et al. (2015), uma relação de canal entre duas empresas poderia ser analisada de acordo com as relações entre dois indivíduos ou entre um indivíduo e uma organização, por exemplo. Para um estudo mais aprofundado e identificação de importantes gaps recentes para pesquisas em contextos B2B, recomenda-se a utilização do estudo de Lilien (2016) como base teórica.

O desenvolvimento das relações B2B, muitas vezes, se baseia em contatos ou vínculos com indivíduos específicos, não com organizações; e o desenvolvimento de relações interpessoais se torna um pré-requisito em relações comerciais (Lian \& Laing, 2007; Dwyer et al., 1987; Wang, 2007). Um dos meios eficientes de aproximação a clientes, e talvez o mais impactante nas trocas interorganizacionais, é a interação social entre os boundary spanners (Ellegaard, 2012; Larentis et al., 2018).

Segundo Lussier e Hall (2018), para construir e manter relacionamentos rentáveis e de longo prazo em mercados B2B, os boundary spanners são incumbidos de adquirir e gerenciar um dos ativos mais importantes da organização, os clientes. Muitas vezes, esses clientes se identificam mais com o boundary spanner das empresas fornecedoras do que com a própria empresa (Ferguson et al., 2005), e a criação de laços incorporados ao relacionamento interpessoal levam a uma confiança pessoal, útil para iniciar e concluir transações (Dong et al., 2017). Um modelo a ser considerado na pesquisa do papel das relações interpessoais em mercados B2B é 0 modelo de interação do Industrial Marketing and Purchasing Group (IMP; Håkansson, 1982), que considera que o estabelecimento de relacionamentos de longo prazo se baseia nas trocas realizadas por uma série limitada de atores.

A pesquisa sobre as relações interpessoais foi estudada, tradicionalmente, pela psicologia social (Ring \& Van De Ven, 1994; Lambe, Wittmann \& Spekman, 2001; Ellegaard, 2012) e um dos conceitos mais utilizados na previsão de comportamentos e atitudes dos indivíduos são os valores pessoais (Torres et al., 2016; Lichtenstein, Lichtenstein \& Higgs, 2017; Bruni-Bossio, 2018). A Teoria dos Valores Humanos de Schwartz (1992) é uma das correntes mais consolidadas no tema, e vem sendo testada em novos contextos, comprovando-se o poder preditivo dos valores sob diversas circunstâncias (Grueso-Hinestroza \& Antón-Rubio, 2015; Torres et al., 2016). Como o PVQ-RR é a escala mais recente de Schwartz et al. (2012), já foi testada no Brasil por Torres et al. (2016) e sua validação em diferentes contextos foi recomendada pelo próprio autor (Schwartz, comunicação pessoal, 2018). $O$ uso desta é altamente indicada em estudos que procurem avaliar valores humanos pessoais, bem como a congruência entre estes em diferentes amostras, tanto em mercados B2B como B2C.

Sobre a congruência de valores, Wang e Zhang (2017) reconhecem que um padrão de valores e crenças compartilhados ajuda os indivíduos a entender o funcionamento organizacional, apesar de terem considerado a congruência de valores organizacionais, analisando apenas um lado da díade, e não entre indivíduos pertencentes a diferentes organizações. Já o modelo formulado no estudo de Zhang e Bloemer (2008) é o mais indicado para se tomar como base metodológica em pesquisas que utilizem escalas de valores humanos de Schwartz, mesmo que tenha sido aplicada a consumidores finais em contextos B2C, já que este comprova o impacto direto da congruência dos valores de Schwartz (1992) na satisfação.

Enfim, para Kashyap e Sivadas (2012), deve-se levar em consideração a força da satisfação em um relacionamento ao examinar os valores e, por isso, foram formuladas as hipóteses seguintes baseadas em pesquisas que confirmaram a relação positiva da satisfação tanto com confiança e comprometimento (Morgan \& 
Hunt, 1994; Svensson et al., 2010; Voldnes et al., 2012; Mpinganjira et al., 2014; Sarmento et al., 2014; Hashim \& Tan, 2015; Mpinganjira et al., 2017), quanto com a lealdade do cliente empresarial ou consumidor final (Rauyruen \& Miller, 2007; Blocker, Flint, Myers \& Slater, 2011; Pan, Sheng \& Xie, 2012), tornando a satisfação uma ponte de ligação entre importantes variáveis de medida de sucesso em relacionamentos B2B.

Assim como para outros autores de relacionamento B2B, recomenda-se que a satisfação não seja reduzida a uma única dimensão, já que esta pode se manifestar por vários motivos envolvidos em uma relação entre empresas (Sivadas et al., 2012; Voldnes et al., 2012; Dadzie, 2015). Em resumo, pode-se considerar duas principais correntes de estudo: satisfação econômica vs. não econômica (Farrelly \& Quester, 2005; Ferro et al., 2016; Mpinganjira et al., 2017) e satisfação econômica vs. social (Lai, 2007; Geyskens \& Steenkamp, 2000; Ha et al., 2016), que, de forma mais abrangente, podem ser unificadas, integrando-se itens igualmente importantes ao considerar-se a segunda dimensão como satisfação não econômica ou social.

Com o objetivo de se avançar na teoria sobre o tema proposto, por meio da compreensão da relação entre as variáveis apresentadas, o modelo teórico proposto é apresentado a seguir, incluindo os respectivos autores sugeridos como base para a construção do conhecimento ao longo da cadeia (Figura 3).

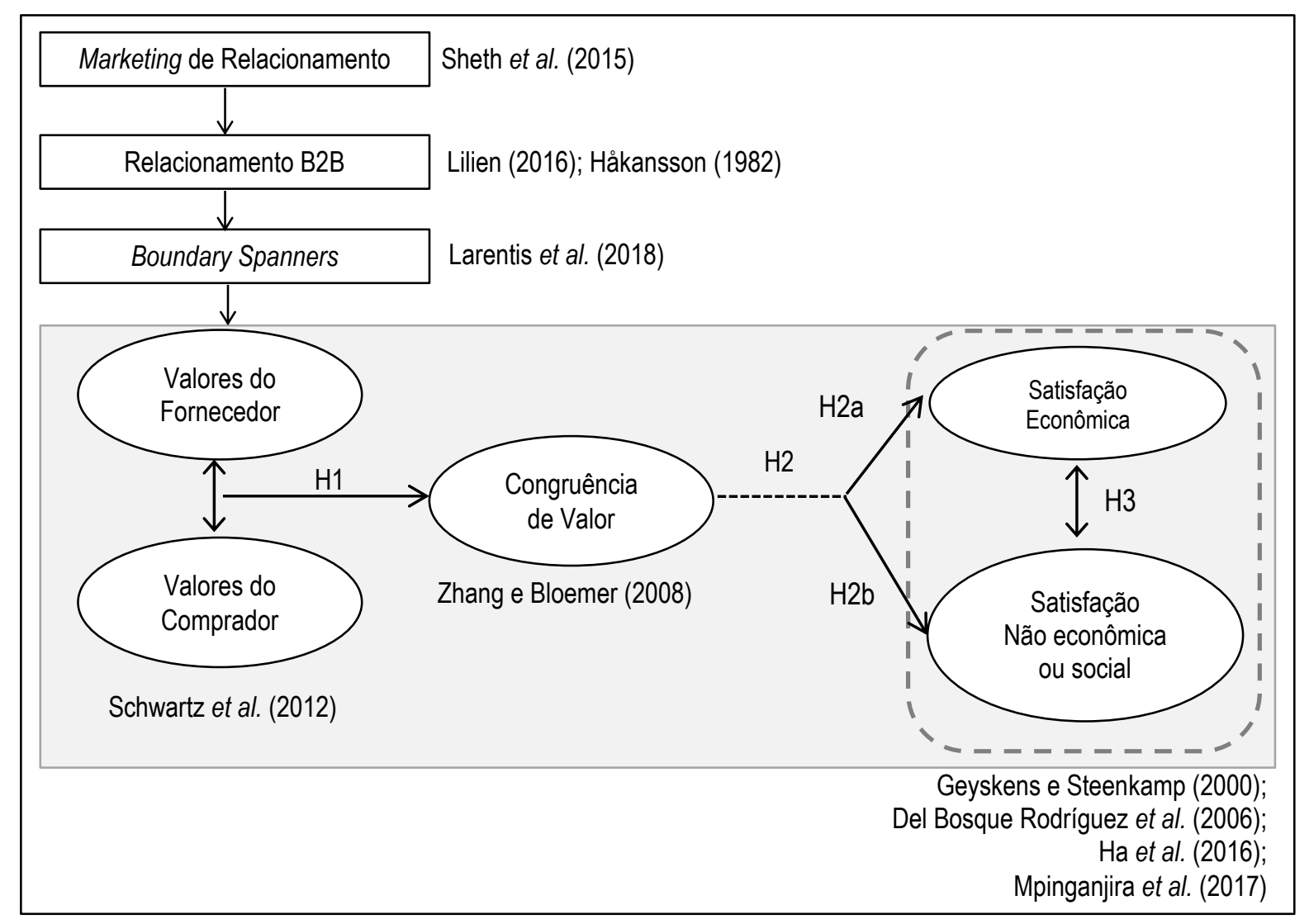

Figura 3: Modelo teórico a ser testado

Fonte: Elaborada pelos autores.

O modelo pode ser aplicado em empresas envolvidas em relações comerciais, tanto de uma perspectiva de díade como de rede. Essas relações podem envolver tanto as díades de fabricante vs. distribuidor ou distribuidor vs. varejista como o canal comercial, ou seja, fabricante vs. distribuidor vs. varejista. O modelo também cabe em estudos sobre as relações estabelecidas com empresas representadas por indivíduos, como médicos, dentistas, agricultores e outros (Lilien, 2016). Como forma de operacionalização, sugerem-se estudos quantitativos, que façam uso de análises estatísticas apropriadas para que cada hipótese possa ser validada.

É recomendado por Schwartz (1992), para avaliação dos valores humanos, o uso da técnica de escalonamento multidimensional, que permite a representação gráfica das correlações entre itens, de forma que quanto mais próximos dois pontos, mais correlacionados positivamente eles estão, e quanto mais distantes, mais eles se correlacionam negativamente. Assim, a análise do gráfico gerado a partir do escalonamento possibilita a 
identificação das dimensões em que os valores estão localizados. Sugere-se também o uso do tratamento Proxscal, um processo que, segundo Sambiase et al. (2014, p. 733), "é realizado através da atribuição de observações em locais específicos num espaço conceitual multidimensional, de tal forma que as distâncias entre os pontos no espaço coincidem com as semelhanças dos dados, neste caso, tanto quanto possível". Além disso, sugere-se que sejam realizadas transformações ordinais de proximidade, e como medida de dissimilaridade a distância euclidiana e a transformação de dados em escores-Z (Torres et al., 2016).

A comparação entre os valores das amostras dos boundary spanners do fornecedor e dos compradores pode ser feita por testes de comparação entre amostras, a fim de verificar a existência de diferenças estatisticamente significativas entre as médias. Para tanto, podem ser utilizadas análises de variância (ANOVA) (Malhotra, 2012). Recomenda-se fortemente que, para o teste do modelo final, que integra o impacto da congruência de valor sobre a satisfação, bem como a relação entre a satisfação econômica e não econômica, seja realizado o procedimento de modelagem de equação estrutural (MEE), já que não se sabe exatamente a relação de dependência entre as variáveis (Hair, Black, Babin, Anderson \& Tatham, 2006). A MEE pode ajudar a avaliar as propriedades de mensuração e testar as relações teóricas propostas utilizando uma única técnica (Malhotra, 2012). Uma variável dependente hipotetizada pode tornar-se uma variável independente em uma relação subsequente de dependência. Além disso, dentro da MEE, é realizada a análise fatorial confirmatória que, segundo Laros (2012), é particularmente útil em estudos psicológicos, que envolvem escalas para medir personalidade, comportamentos e atitudes, por testar a unidimensionalidade dos itens para um único construto.

\section{CONSIDERAÇÕES FINAIS}

O objetivo do estudo foi alcançado por meio da apresentação do modelo teórico desenvolvido, que propõe a avaliação do impacto da congruência dos valores humanos dos boundary spanners na satisfação dos membros envolvidos em relações interorganizacionais. As hipóteses formuladas, a partir de uma robusta pesquisa teórica realizada, permitem prever relações entre os construtos apresentados, de forma que resultados de futuros estudos contribuirão com o avanço do conhecimento das temáticas.

A averiguação empírica do modelo poderá elucidar alguns aspectos obscuros do comportamento de compra organizacional, que ainda permanecem sem resposta. De uma perspectiva prática, os valores, por serem muito intangíveis, muitas vezes não são considerados no momento da escolha do parceiro comercial, o que pode se tornar um problema a longo prazo. De uma perspectiva teórica, a miopia sobre a psicologia das relações interorganizacionais se dá tanto por parte dos gestores quanto dos psicólogos (Schruijer, 2014).

Mesmo que haja muitas pesquisas recentes que continuam estudando os relacionamentos B2B (Bocconcelli, Murmura, \& Pagano, 2018; Liu, Deligonul, Cavusgil \& Chiou, 2018; Lussier \& Hall, 2018), assim como os valores humanos pessoais (Torres et al., 2016; Ahmad \& Omar, 2018; Frendika, Sule, Kusman \& Joeliaty, 2018) ou a satisfação (Ferro et al., 2016; Ha et al., 2016; Mpinganjira et al., 2017), poucos estudiosos abordam esses temas de forma unificada (Ellegaard, 2012; Kashyap \& Sivadas, 2012; Bawa, 2016), conforme foi apresentado.

Espera-se, a partir do modelo proposto, que estudos futuros possam preencher essas lacunas, e que outras variáveis relevantes possam ser inseridas gradativamente como mediadoras. A confiança e 0 comprometimento, por exemplo, já foram relacionados com a congruência de valores (Dwyer et al., 1987; Sitkin \& Roth, 1993; Morgan \& Hunt, 1994; Ren, 2010) e com a satisfação (Svensson et al., 2010; Voldnes et al., 2012; Mpinganjira et al., 2014; Sarmento et al., 2014; Hashim \& Tan, 2015; Mpinganjira et al., 2017), mas não foram encontrados, ao longo da pesquisa realizada, estudos que avaliem o potencial mediador desses elementos entre a congruência de valores e a satisfação. Esta seria uma via alternativa de pesquisa sobre o tema.

\section{REFERÊNCIAS}

Abdul-Muhmin, A. G. (2005). Instrumental and interpersonal determinants of relationship satisfaction and commitment in industrial markets. Journal of Business Research, 58, 619-628.

Ahmad, S.N.B. \& Omar, A. (2018). Influence of perceived value and personal values on consumers repurchase intention of natural beauty product. International Journal of Supply Chain Management, 7(2), 116-125.

Ajzen, I. (1971). Attribution of dispositions to an actor: effects of perceived decision freedom and behavioral utilities. Journal of Personality and Social Psychology, 18(2), 144-156.

Revista Alcance - Eletrônica - vol. 25 - n. 2 - Mai./Ago. 2018 
Andrade, M. L. \& Leite, R. S. (2017). Teoria dos valores pessoais em marketing: um estudo bibliométrico sobre as publicações nacionais. Anais do Encontro Nacional da Associação Nacional de Programas de PósGraduação em Administração - EnANPAD, São Paulo, SP, Brasil, 42. Recuperado de http://www.anpad.org.br/ anpad/abrir_pdf.php?e=MjM4MTA=

Araújo, C. A. (2002). Bibliometria: evolução histórica e questões atuais. Em Questão, 12(1), 11-32.

Araujo, L., Dubois, A. \& Gadde, L. (2003). The multiple boundaries of the firm. Journal of Management Studies, 40(5), 1255-1277.

Bawa, P. (2016). How value congruence affect customer focus and survival of business? International Journal of Applied Business and Economic Research, 14(7), 5085-5096.

Beechler, S., Sondergaard, M., Miller, E. \& Bird, A. (2004). Boundary Spanning. In H. Lane, M. Maznevski, M. Mendenhall \& J. McNett (Eds.). The Handbook of Global Management: A Guide to Managing Complexity (pp. 121-133). Malden: Blackwell Publishing.

Berry, L. L. (1983). Relationship marketing. In L. L. Berry, G. L. Shostack \& G. Upah (Eds.). Emerging perspectives on services marketing (pp. 25-28). Chicago: American Marketing Association.

BLAU, P. M. (1964). Exchange and power in social life. New Brunswick: Transaction.

Blocker, C. P., Flint, D. J., Myers, M. B. \& Slater, S. F. (2011). Proactive customer orientation and its role for creating customer value in global markets. Journal of the Academy of Marketing Science, 39(2), 216-233.

Bocconcelli, R., Murmura, F. \& Pagano, A. (2018). Interacting with large customers: Resource development in small b2b suppliers. Industrial Marketing Management, 70, 101-112.

Bruni-Bossio, V. (2018). Corporate board decision-making: applying collective versus personal values. Journal of Business Strategy, 39(1), 15-23.

Byza, O. A. U., Dörr, S. L., Schuh, S. C. \& Maier, G. W. (2017). When leaders and followers match: the impact of objective value congruence, value extremity, and empowerment on employee commitment and job satisfaction. Journal of Business Ethics, 1-16.

Cazier, J., Shao, B. \& Louis, R. St. (2017). Value congruence, trust, and their effects on purchase intention and reservation price. ACM Transactions on Management Information Systems, 8(4), 1-28.

Chakkol, M., Karatzas, A., Johnson, M. \& Godsell, J. (2018). Building bridges: boundary spanners in servitized supply chains. International Journal of Operations \& Production Management, 38(2), 579-604.

Cieciuch, J., Schwartz, S.H. \& Davidov, E. (2015). Social psychology of values. In J. D. WRIGHT (Ed.). International encyclopedia of the social \& behavioral sciences (2a ed., v. 25, pp. 41-46). Oxford: Elsevier.

Cortez, R. M. \& Johnston, W. J. (2017). The future of B2B marketing theory: a historical and prospective analysis. Industrial Marketing Management, 66, 90-102.

Coughlan, A T., Anderson, E., Stern, L. W. \& El-Ansary, A. I. (2012). Canais de Marketing (7a ed). São Paulo: Pearson Education do Brasil.

Cropper, S., Ebers, M., Huxham, C., \& Ring, P. S. (2014). Introdução às relações interorganizacionais. In S. Cropper, M. Ebers, C. Huxham, \& P. S. Ring (Eds.). Handbook de relações interorganizacionais da Oxford (pp. 3-20). Porto Alegre: Bookman.

Dadzie, C. A. (2015). Channel member satisfaction and performance in african export marketing channels: some insights from Ghana. Journal of Marketing Channels, 22(2), 108-120.

Del Bosque Rodríguez, I. R., Agudo, J. C. \& San Martín Gutiérrez, H. (2006). Determinants of economic and social satisfaction in manufacturer-distributor relationships. Industrial Marketing Management, 35(6), 666-675.

Dong, W., Ma, Z. \& Zhou, X. (2017). Relational governance in supplier-buyer relationships: the mediating effects of boundary spanners' interpersonal guanxi in China's B2B market. Journal of Business Research Relational, 78, 332- 340.

Dwyer, F. R. (1980). Channel-member satisfaction: laboratory insights. Journal of Retailing, 56(2), 45-65. 
Dwyer, F. R., Schurr, P. H. \& Oh, S. (1987). Developing buyer-seller relationships. Journal of Marketing, 51(2), $11-27$.

Eagly, A.H. \& Wood, W. (1999). The origins of sex differences in human behavior: Evolved dispositions versus social roles. American Psychologist, 54(6), 408-423.

Ellegaard, C. (2012). Interpersonal attraction in buyer-supplier relationships: a cyclical model rooted in social psychology. Industrial Marketing Management, 41(8), 1219-1227.

Enz, C. A. (1988). The role of value congruity in intraorganizational power. Administrative Science Quarterly, 33, 284-304.

Eva, N., Prajogo, D. \& Cooper, B. (2017). The relationship between personal values, organizational formalization and employee work outcomes of compliance and innovation. International Journal of Manpower, 38(2), 274287.

Farrelly, F. J. \& Quester, P. G. (2005). Examining important relationship quality constructs of the focal sponsorship exchange. Industrial Marketing Management, 34(3), 311-319.

Ferguson, R. J., Paulin, M. \& Bergeron, J. (2005). Contractual Governance, Relational governance, and the performance of interfirm service exchanges: the influence of boundary- spanner closeness. Journal of the Academy of Marketing Science, 33(2), 217-234.

Ferro, C., Padin, C., Svensson, G. \& Payan, J. (2016). Trust and commitment as mediators between economic and non-economic satisfaction in manufacturer-supplier relationships. Journal of Business and Industrial Marketing, 31(1), 13-23.

Frendika, R., Sule, E.T., Kusman, M. \& Joeliaty. (2018). The power of personal values and cultural competence towards personal branding of employees. Academy of Strategic Management Journal, 17(1), 1-10.

Fula, M. (2004). The problem of happiness in moral philosophy. Filozofia, 59(2), 110-123.

Gaunt, R. (2006). Couple similarity and marital satisfaction: are similar spouses happier? Journal of Personality, 74(5), 1401-1420.

Geyskens, I. \& Steenkamp, J.-B. E. M. (2000). Economic and social satisfaction: measurement and relevance to marketing channel relationships. Journal of Retailing, 76(1), 11-32.

Geyskens, I., Steenkamp, J.-B. E. M., \& Kumar, N. (1999). A meta-analysis of satisfaction in marketing channel relationships. Journal of Marketing Research, 36(2), 223-238.

González-Rodríguez, M. R., Díaz-Fernández, M. C., \& Simonetti, B. (2015). The social, economic and environmental dimensions of corporate social responsibility: the role played by consumers and potential entrepreneurs. International Business Review, 24, 836-848.

Grewal, R., Lilien, G. L., Bharadwaj, S., Jindal, P., Kayande, U., Lusch, R. F., Mantrala, M., Palmatier, R. W., Rindfleisch, A., Scheer, L. K., Spekman, R. \& Sridhar, S. (2015). Business-to-Business buying: challenges and opportunities. Customer Needs and Solutions, 2(3), 193-208.

Grönroos, C. (2004). The relationship marketing process: communication, interaction, dialogue, value. Journal of Business \& Industrial Marketing, 19(2), 99-113.

Grueso-Hinestroza, M. P., \& Antón-Rubio, M. C. (2015). Cultural values in interorganizational networks: a proposal from the model of Shalom Schwartz (1992). Investigación y Desarrollo, 23(2), 369-390.

Ha, H.-Y., Lee, M.-S \& Janda, S. (2016). Effects of economic and social satisfaction on partner trust: an investigation of temporal carryover effects. European Journal of Marketing, 50(1/2), 100-123.

Hair, J. F., Black, W. C., Babin, B. J., Anderson, R. E., \& Tatham, R. L. (2006). Multivariate data analysis. Upper Saddle River: Pearson Prentice Hall.

Håkansson, H. (1982). International marketing and purchasing of industrial goods: an interaction approach. Chichester: Wiley. 
Harmeling, C. \& Palmatier, R. W. (2015). Relationship dynamics: understanding continuous and discontinuous relationship change. In C. A. INGENE, \& R. P. DANT (Eds.). Handbook of research on distribution channels (pp. 1-32). Northampton: Edward Elgar Publishing.

Hashim, K. F. \& Tan, F. B. (2015). The mediating role of trust and commitment on members' continuous knowledge sharing intention: a commitment-trust theory perspective. International Journal of Information Management, 35(2), 145-151.

He, J., Huang, H. \& Wu, W. (2018). Influence of interfirm brand values congruence on relationship qualities in B2B contexts. Industrial Marketing Management, 72, 161-173.

Heide, J. B. \& John, G. (1992). Do norms matter in marketing relationships? Journal of Marketing, 56, 32-44.

Human, G. \& Naudé, P. (2014). Heterogeneity in the quality-satisfaction-loyalty framework. Industrial Marketing Management, 43(6), 920-928.

Jensen, U. T. (2018). Does perceived societal impact moderate the effect of transformational leadership on value congruence? Evidence from a field experiment. Public Administration Review, 78(1), 48-57.

Johnsen, T. E., Lamming, R. C. \& Harland, C. M. (2014). Relacionamentos interorganizacionais, cadeias e redes: uma perspectiva de suprimentos. In: S. Cropper, M. Ebers, C. Huxham \& P. S. Ring (Eds.). Handbook de relações interorganizacionais da Oxford (pp. 57-81). Porto Alegre: Bookman.

Kashyap, V. \& Sivadas, E. (2012). An exploratory examination of shared values in channel relationships. Journal of Business Research, 65(5), 586-593.

Lai, C. -S. (2007). The effects of influence strategies on dealer satisfaction and performance in Taiwan's motor industry. Industrial Marketing Management, 36, 518-527.

Lajoie, D., Boudrias, J.-S., Rousseau, V. \& Brunelle, É. (2017). Value congruence and tenure as moderators of transformational leadership effects. Leadership and Organization Development Journal, 38(2), 254-269.

Lambe, C. J.; Wittmann, C. M.; Spekman, R. E. (2001). Social exchange theory and research on business-tobusiness relational exchange. Journal of Business-to-Business Marketing, 8(3), 1-36.

Larentis, F., Antonello, C. S. \& Slongo, L. A. (2018). Cultura organizacional e marketing de relacionamento: uma perspectiva interorganizacional. Revista Brasileira de Gestão de Negócios, 20(1), 37-56.

Larentis, F. \& Slongo, L. A. (2008). Relacionamento em canais de marketing como fonte de vantagem competitiva sustentável: um estudo com fabricantes de móveis e lojas exclusivas. Revista de Administração da USP, 43(30), 209-223.

Laros, J. A. (2012). O uso da análise fatorial: algumas diretrizes para pesquisadores. In L. PASQUALI (Org.). Análise fatorial para pesquisadores (pp. 141-160). Brasilia: LabPAM.

Lebbon, A. R. \& Sigurdsson, S. O. (2017). Behavioral perspectives on variability in human behavior as part of process safety. Journal of Organizational Behavior Management, 37(3-4), 261-282.

Lee, J., Sneddon, J. N., Daly, T. M, Schwartz, S. H., Soutar, G. N. \& Louviere, J. J. (2017). Testing and extending Schwartz refined value theory using a best-worst scaling approach. Assessment, 1-15.

Leung, T. K. P., Lai, K., Chan, R. Y. K., \& Wong, Y. H. (2005). The roles of xinyong and guanxi in Chinese relationship marketing. European Journal of Marketing, 39(5/6), 528-565.

Lian, P. C. S. \& Laing, A. W. (2007). Relationships in the purchasing of business to business professional services: The role of personal relationships. Industrial Marketing Management, 36, 709-718.

Lichtenstein, S., Lichtenstein, G. \& Higgs, M. (2017). Personal values at work: A mixed-methods study of executives' strategic decision-making. Journal of General Management, 43(1), 15-23.

Lilien, G. L. (2016). The B2B knowledge gap. International Journal of Research in Marketing, 33, 543-556.

Liu, Y. H.-S., Deligonul, S., Cavusgil, E. \& Chiou, J.-S. (2018). Always trust in old friends? Effects of reciprocity in bilateral asset specificity on trust in international B2B partnerships. Journal of Business Research, 90, 171 185. 
Lussier, B. \& Hall, Z. R. (2018). Cooperation in B2B relationships: factors that influence customers' perceptions of salesperson cooperation. Industrial Marketing Management, 69, 209-220.

Lussier, B. \& Hartmann, N. (2017). How psychological resourcefulness increases salesperson's sales performance and the satisfaction of their customers: Exploring the mediating role of customer-oriented behaviors. Industrial Marketing Management, 62, 160-170.

Lyons, P., Wodarski, J. S. \& Feit, M. D. (1998). Human behavior theory: Emerging trends and issues. Journal of Human Behavior in the Social Environment, 1(1), 1-21.

Madi, A. (2016). Using values to segment virtual consumers on social networking sites. Marketing Intelligence \& Planning, 34(5), 623-645.

Malbašić, I., Rey, C. \& Potočan, V. (2015). Balanced organizational values: from theory to practice. Journal of Business Ethics, 130(2), 437-446.

Malhotra, N. K. (2012). Pesquisa de marketing: uma orientação aplicada (6a ed.). Porto Alegre: Bookman.

Mehta, R., Larsen, T., Rosenbloom, B. \& Ganitsky, J. (2006). The impact of cultural differences in U.S. businessto-business export marketing channel strategic alliances. Industrial Marketing Management, 35, 156-165.

Moorman, C., Zaltman, G. \& Deshpandé, R. (1992). Relationships between providers and users of market research: the dynamics of trust within and between organizations. Journal of Marketing Research, 29, 314-328.

Morgan, R. M. \& Hunt, S. D. (1994). The commitment-trust theory of relationship marketing. Journal of Marketing, 58(3), 20-38.

Mpinganjira, M., Bogaards, M., Svensson, G. \& Mysen, T. (2014). Precursors and outcomes of satisfaction in business relationships: a replication study. Management Dynamics, 23(2), 2-13.

Mpinganjira, M., Roberts-Lombard, M. \& Svensson, G. (2017). Validating the relationship between trust, commitment, economic and non-economic satisfaction in South African buyer-supplier relationships. Journal of Business \& Industrial Marketing, 32(3), 421-431.

Murry, J. P. J. \& Heide, J. B. (1998). Managing promotion program participation within manufacturer-retailer relationships. Journal of Marketing, 62(1), 58-68.

Pan, Y., Sheng, S. \& Xie, F. T. (2012). Antecedents of customer loyalty: an empirical synthesis and reexamination. Journal of Retailing and Consumer Services, 19(1), 150-158.

Paswan, A. K., Blankson, C. \& Guzman, F. (2011). Relationalism in marketing channels and marketing strategy. European Journal of Marketing, 45(3), 311-333.

Pavlov, I. P. \& Gantt, W. H. (1941). Conditioned reflexes and psychiatry. New York: International publishers.

Porto, J. B. (2005). Mensuração de valores no Brasil. In A. TAMAYO, \& J. B. PORTO (Eds.). Valores e comportamentos nas organizações (pp. 93-119). Petrópolis: Vozes.

Qu, Y. E., Dasborough, M. T., Zhou, M. \& Todorova, G. (2017). Should authentic leaders value power? A study of leaders' values and perceived value congruence. Journal of Business Ethics, 1-18.

Ramaseshan, B., Yip, L. S. C. \& Pae, J. H. (2006). Power, satisfaction, and relationship commitment in Chinese store-tenant relationship and their impact on performance. Journal of Retailing, 82(1), 63-70.

Rauyruen, P. \& Miller, K. E. (2007). Relationship quality as a predictor of B2B customer loyalty. Journal of Business Research, 60(1), 21-31.

Rein, M. \& Eysenck, H. (2018). Dimensions of Personality. Routledge.

Ren, T. (2010). Value congruence as a source of intrinsic motivation. Kyklos, 63(1), 94-109.

Ring, P. S. \& Van De Ven, A. H. (1994). Developmental processes of cooperative interorganizational relationships. Academy of Management Review, 19(1), 90-118.

Rokeach, M. (1973). The nature of human values. New York: Free Press.

Sabbir, R. M. \& Nazrul, I. (2014). Value congruence and consumer's satisfaction towards online banking - The mediation role of affective commitment. Management and Marketing, 9(3), 347-358.

Revista Alcance - Eletrônica - vol. 25 - n. 2 - Mai./Ago. 2018 
Sambiase, M. F., Teixeira, M. L. M., Bilsky, W., Araujo, B. F. \& Domenico, S. M. R. (2014). Confrontando estruturas de valores: um estudo comparativo entre PVQ-40 e PVQ-21. Psicologia: Reflexão e Crítica, 27(4), 728-739.

Sarmento, M., Simões, C. \& Farhangmehr, M. (2014). Applying a relationship marketing perspective to B2B trade fairs: the role of socialization episodes. Industrial Marketing Management, 44(1), 131-141.

Schein, E. H. (2010). Organizational culture and leadership (6a ed.). San Francisco: Wiley.

Schruijer, S. G. L. (2014). A psicologia social das relações interorganizacionais. In S. Cropper, M. Ebers, C. Huxham \& P. S. Ring (Eds.). Handbook de relações interorganizacionais da Oxford (pp. 376-397). Porto Alegre: Bookman

Schwartz, S. H. (1992). Universals in the content and structure of values: theoretical advances and empirical tests in 20 countries. Advances in Experimental Social Psychology, 25, 1-65.

Schwartz, S. H. (2005). Valores humanos básicos: Seu contexto e estrutura intercultural. In A. Tamayo, \& J. B. PORTO (Eds.). Valores e comportamentos nas organizações (pp. 17-55). Petrópolis: Vozes.

Schwartz, S. H. \& Bilsky, W. (1987). Toward a universal psychological structure of human values. Journal of Personality and Social Psychology, 53(3), 550-562.

Schwartz, S. H., Cieciuch, J., Vecchione, M., Davidov, E., Fischer, R., Beierlein, C., Ramos, A., Verkasalo, M., Lönnqvist, J.-E., Demirutku, K., Dirilen-Gumus, O. \& Konty, M. (2012). Refining the theory of basic individual values. Journal of Personality and Social Psychology, 103(4), 663-688.

Schwartz, S. H., Melech, G., Lehmann, A., Burgess, S., Harris, M. \& Owens, V. (2001). Extending the CrossCultural Validity of the Theory of Basic Human Values with a Different Method of Measurement. Journal of Cross-Cultural Psychology, 32(5), 519-542.

Seabright, M. A., Levinthal, D. A. \& Fichman, M. (1992). Role of individual attachments in the dissolution of interorganizational relationships. The Academy of Management Journal, 35(1), 122-160.

Shani, D. \& Chalasani, S. (1992). Exploiting niches using relationship marketing. Journal of Consumer Marketing, 9(3), 33-42.

Sheth, J.N., Parvatiyar, A. \& Sinha, M. (2015). The conceptual foundations of relationship marketing: review and synthesis. Journal of Economic Sociology, 16(2), 119-149.

Sitkin, S. B. \& Roth, N. L. (1993). Explaining the limited effectiveness of legalistic "remedies" for trust/distrust. Organization Science, 4(3), 367-392.

Sivadas, E., Holmes, T.L. \& Dwyer, F.R. (2012). Interorganizational information systems and business-to-business relationships: system characteristics, assistance, performance, satisfaction, and commitment model. Journal of Marketing Channels, 19(1), 17-48.

Splitter, K., Rosa, C. A. \& Borba, J. A. (2012). Uma Análise das Características dos Trabalhos "Ditos" Bibliométricos Publicados no Enanpad entre 2000 e 2011. Anais do Encontro Nacional da Associação Nacional de Programas de Pós-Graduação em Administração - EnANPAD, São Paulo, SP, Brasil, 36. Recuperado de http://www.anpad.org.br/admin/pdf/2012_EPQ2501.pdf

Svensson, G., Mysen, T. \& Payan, J. (2010). Balancing the sequential logic of quality constructs in manufacturingsupplier relationships - Causes and outcomes. Journal of Business Research, 63(11), 1209-1214.

Tamayo, A. (2007). Contribuições ao estudo dos valores pessoais, laborais e organizacionais. Psicologia: Teoria e Pesquisa, 23, 17-24.

Tamayo, A. (2005). Impacto dos valores pessoais e organizacionais sobre o comprometimento organizacional. In A. Tamayo \& J. B. Porto (Eds.). Valores e comportamentos nas organizações. Petrópolis: Vozes.

Teixeira, M. L. M., Iwamoto, H. M. \& Medeiros, A. L. (2013). Estudos bibliométricos(?) em administração: discutindo a transposição de finalidade. Administração: ensino e pesquisa, 14(3), 423-452.

Thøgersen, J. \& Olander, F. (2002). Human values and the emergence of a sustainable consumption pattern: a panel study. Journal of Economic Psychology, 23, 605-630. 
Torres, C. V., Schwartz, S. H. \& Nascimento, T. G. (2016). A teoria de valores refinada: associações com comportamento e evidências de validade discriminante e preditiva. Psicologia USP, 27(2), 341-356.

Valério, F. (2015). Práticas de marketing de relacionamento entre fabricantes e distribuidores de defensivos agrícolas (Dissertação de Mestrado). Universidade de São Paulo, Ribeirão Preto, SP, Brasil. Recuperado de http://www.teses.usp.br/teses/disponiveis/96/96132/tde-02122015-165757/pt-br.php

Vilela, T. S. V. (2017). Valores humanos pessoais predizem comportamento ecológico? (Dissertação de Mestrado). Universidade de Brasília, Brasília, DF, Brasil. Recuperado de http://repositorio.unb.br/bitstream/10482/24272/1/2017_ThâmaraSampaioVasconcelosVilela.pdf

Voldnes, G., Grønhaug, K. \& Nilssen, F. (2012). Satisfaction in buyer-seller relationships-influence of cultural differences. Industrial Marketing Management, 41(7), 1081-1093.

Wang, B., Kang, Y., Childerhouse, P. \& Huo, B. (2018). Service supply chain integration: the role of interpersonal relationships. Industrial Management \& Data Systems, 118(4), 828-849.

Wang, C. L. (2007). Guanxi vs. relationship marketing: exploring underlying differences. Industrial Marketing Management, 36, 81-86.

Wang, J. J. \& Zhang, C. (2017). The impact of value congruence on marketing channel relationship. Industrial Marketing Management, 62, 118-127.

Watson, G. F., Worm, S., Palmatier, R. W. \& Ganesan, S. (2015). The evolution of marketing channels: trends and research directions. Journal of Retailing, 91(4), 546-568.

Webster, F. E. \& Keller, K. L. (2004). A roadmap for branding in Industrial Markets. Brand Management, 11(5), 388402.

Webster, F. E. \& Wind, Y. (1996). A general model for understanding organizational buying behavior. Marketing Management, 4(4), 52-57.

Woodruff, A. D. (1949). The concept-value theory of human behavior. Journal of General Psychology, 40(2), 141154.

Ye, S., Soutar, G. N., Sneddon, J. N. \& Lee, J. A. (2017). Personal values and the theory of planned behaviour: A study of values and holiday trade-offs in young adults. Tourism Management, 62, 107-109.

Yeung, M.C., Ramasamy, B., Chen, J. \& Paliwoda, S. (2013). Customer satisfaction and consumer expenditure in selected European countries. International Journal of Research in Marketing, 30(4), 406-416.

Zhang, J. Z., Netzer, O. \& Ansari, A. (2014). Dynamic targeted pricing in B2B relationships. Marketing Science, 33, 317-337.

Zhang, J. Z., Watson, G. F., Palmatier, R. W. \& Dant, R. P. (2016). Dynamic relationship marketing. Journal of Marketing, 80(5), 53-75.

Zhang, J. \& Bloemer, J. M. M. (2008). The impact of shared values on consumer-service brand relationships. Journal of Service Research, 11(2), 161-78.

Zhu, D. H. \& Chang, Y. P. (2013). Negative publicity effect of the business founder's unethical behaviour on corporate image: evidence from China. Journal Business Ethics, 117, 111-121.

Zhuang, G. \& Zhang, X. (2011). Impact of relationship marketing orientation on the exercise of interfirm power and relational governance in marketing channels: empirical evidence from China. Journal of Marketing Channels, 18(4), 279-302. 